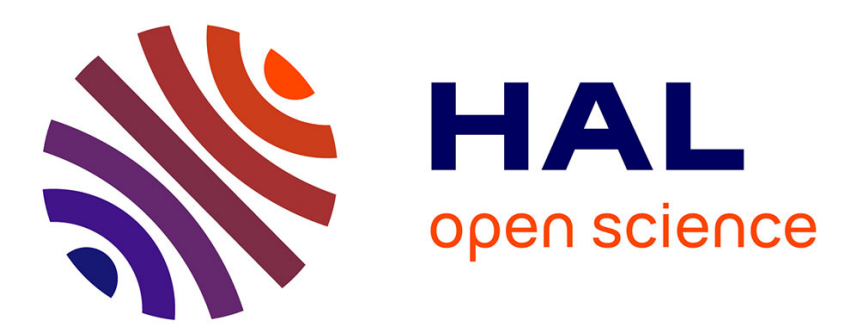

\title{
Joint estimation of channel and carrier frequency offset from the emitter, in an uplink OFDMA system
}

Babar Aziz, Inbar Fijalkow, Myriam Ariaudo

\section{To cite this version:}

Babar Aziz, Inbar Fijalkow, Myriam Ariaudo. Joint estimation of channel and carrier frequency offset from the emitter, in an uplink OFDMA system. IEEE International Conference on Acoustics, Speech and Signal Processing (ICASSP), 2011, May 2011, Prague, Czech Republic. pp.3492 - 3495, 10.1109/ICASSP.2011.5946230 . hal-00649412

\section{HAL Id: hal-00649412 \\ https://hal.science/hal-00649412}

Submitted on 7 Dec 2011

HAL is a multi-disciplinary open access archive for the deposit and dissemination of scientific research documents, whether they are published or not. The documents may come from teaching and research institutions in France or abroad, or from public or private research centers.
L'archive ouverte pluridisciplinaire HAL, est destinée au dépôt et à la diffusion de documents scientifiques de niveau recherche, publiés ou non, émanant des établissements d'enseignement et de recherche français ou étrangers, des laboratoires publics ou privés. 


\title{
JOINT ESTIMATION OF CHANNEL AND CARRIER FREQUENCY OFFSET FROM THE EMITTER, IN AN UPLINK OFDMA SYSTEM
}

\author{
Babar Aziz, Inbar Fijalkow and Myriam Ariaudo \\ ETIS, ENSEA, Université de Cergy-Pontoise, CNRS, \\ F-95000 Cergy-Pontoise Cedex, France \\ E-mails: \{babar.aziz,inbar.fijalkow,myriam.ariaudo\}@ensea.fr
}

\begin{abstract}
In this paper, we propose a joint estimation method of carrier frequency offsets (CFO) and channel impulse responses (CIR) for an uplink OFDMA system. We consider the CFOs come into play from the emitters due to frequency mismatch between the terminals and base station oscillators. We also take into account the cyclic prefix to derive a new model of the received signal.
\end{abstract}

Index Terms - Carrier frequency offset, OFDMA uplink transmission, channel estimation, carrier frequency offset estimation

\section{INTRODUCTION}

The new mobile communication systems such as Wifi, WiMAX and LTE (Long Term Evolution) have to offer high data rates both in the uplink and downlink directions in order to fulfill the needs of the innovative multimedia applications $[1,2]$. OFDMA is coming forth as the favored downlink transmission scheme for these systems because it is highly robust in frequency selective radio channels and also provides a good system flexibility. However, questions are still raised about the use of OFDMA in the uplink direction. A well known problem of OFDMA in the uplink is its sensitivity to carrier frequency offsets (CFOs). This paper concentrates on carrier frequency synchronization error while perfect time synchronization is assumed. The frequency mismatch may result from the user terminals oscillators mismatches with the base station oscillator. This phenomenon is all the more important than the terminals must be cheap. The different CFOs can not be canceled at the base station, resulting in severe interference, see [3], which degrades the system performance. The channel estimation for OFDM systems has been well studied, e.g. [4, 5]. However when uplink OFDMA transmission is considered, an efficient joint estimation of the channel impulse responses and CFOs is required in the presence of frequency mismatch. A few authors have addressed this issue

This work is supported by the French IdF Region DIM "Logiciels et Systmes Complexes" Project DESAP. in uplink OFDMA system $[6,7]$. However most of the presented algorithms consider the estimation of the CFO alone without considering the channel estimation. Some studies have been presented in [8, 9]. In [8], joint CFO and channel estimation is done using the maximum-likelihood approach but it proves to be highly complex. In [9], authors have presented the estimator based on a polynomial approximation for the CFO estimation which tends to reduce the complexity one faces in the grid search algorithms. However all the authors while doing the joint estimation for the uplink OFDMA transmission assume that the $\mathrm{CFO}$ is applied to the signal after the channel convolution i.e. at the receiver. Unlike them, we consider a signal model considering that CFO comes strictly from the mismatch of the emitter oscillator with the base station, i.e. before the channel convolution. Therefore the CFO comes into action from the transmitted signal presented at the output of the emitter. Based on this new transmission model we show that our proposed algorithm further simplifies the CFO estimation compared to the approach provided in [9].

In section 2, the considered system model is presented along with an analytical signal model. In section 3 , the proposed joint estimation algorithm is presented followed by the numerical results in Section 4.

\section{UPLINK SYSTEM MODEL: CFO FROM EMITTER MODEL}

In this section, we present the uplink OFDMA system under consideration. The available bandwidth $B$ is divided into $N_{p}$ subcarriers. $N_{u}$ is the total number of users in the system. The radio channel model is assumed to be frequency selective. The last $L_{c p}$ modulation symbols in the OFDM symbol are repeated after the inverse DFT to form the cyclic prefix. The signal at the output of the transmitter of user $u$ is given by

$$
x^{(u)}(t)=\operatorname{Re}\left\{e^{j 2 \pi\left(f_{c}+\delta f_{c}^{(u)}\right) t} \sum_{k=0}^{N_{p}-1} a_{k}^{(u)} p^{(u)}\left(t-\frac{k T}{N_{p}}\right)\right\}
$$

where $f_{c}$ is the carrier frequency, $p^{(u)}(t)$ is the impulse response of the low pass filter at the transmitter and $T$ is the 
symbol period of an OFDMA symbol. $\delta f_{c}^{(u)}$ represents the CFO of user $u$ with respect to $f_{c}$. The CFO appears in the transmitted signal because we are considering the case where the user $u$ terminal is not perfectly synchronized to the carrier frequency of the base station due to the components misbehavior. Note that $a_{k}^{(u)}$ are the symbols at the IDFT output. Signals from different users $x^{(u)}(t)$ pass through individual convolutive multipath radio channels. The received signal at the base station is the sum of the signals from all users. The received discrete-time baseband signal $r_{i}$ at the base station is given by

$$
r_{i}=\sum_{u=1}^{N_{u}}\left\{x_{i}^{(u)} e^{\frac{j 2 \pi}{N_{p}}\left(L_{h}+i-1\right) \delta f^{(u)}}\right\} * h_{i}^{(u)}+n_{i}
$$

where $*$ stands for convolution.

$$
r_{i}=\sum_{u=1}^{N_{u}} \sum_{l=0}^{L_{h}-1} x_{i-l}^{(u)} e^{\frac{j 2 \pi}{N_{p}}\left(L_{h}+i-l-1\right) \delta f^{(u)}} h_{l}^{(u)}+n_{i}
$$

where $x_{i}^{(u)}$ is the $i^{t h}$ sample of the transmitted signal of user $u$ and $h_{l}^{(u)}$ denotes the $l^{t h}$ sample of the impulse response of the channel for user $u$, including the transmitter and receiver filters, $n_{i}$ is the additive white Gaussian noise. $L_{h}$ is the channel length which is assumed to be less than the cyclic prefix length i.e. $L_{h}<L_{c p}$. Note that $\delta f^{(u)}$ is the normalized CFO of user $u$ and is given by

$$
\delta f^{(u)}=\delta f_{c}^{(u)} T
$$

Since we wanted to perform channel and CFO estimation, we carefully worked on eq.(3) to separate the different contributions. By collecting the $N_{p}$ samples and removing the cyclic prefix as in [3], we have derived the following new expression:

$$
\underline{r}=\sum_{u=1}^{N_{u}}\left(\underline{\delta}^{(u)} \odot \underline{\tilde{X}}^{(u)}\right) \underline{h}^{(u)}+\underline{n}
$$

where $\underline{r}$ is an $N_{p} \times 1$ vector containing the samples of the received signal and $\underline{n}$ is the $N_{p} \times 1$ noise vector with noise samples. The $\left(\underline{\delta}^{(u)} \odot \underline{\widetilde{X}}^{(u)}\right)$ product, in eq.(4), is shown below in matrix form, where the index $u$ is removed for simplicity,

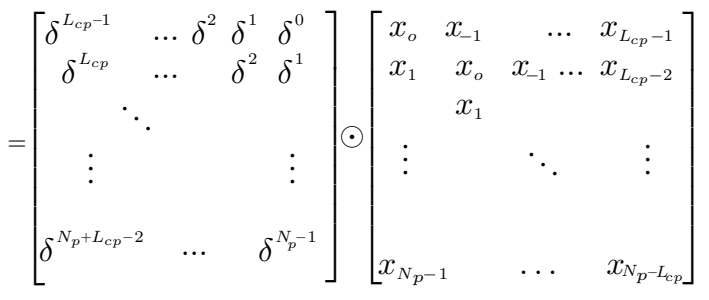

The $N_{p} \times L_{c p}$ matrix $\underline{\widetilde{X}}^{(u)}$ has the samples of the transmitted signal along with the cyclic prefix of length $L_{c p}$. The
$N_{p} \times L_{c p}$ matrix $\underline{\delta}^{(u)}$ is the shift matrix of user $u$ containing its shift coefficients. The $\odot$ represents the Hadamard product. Hadamard product comes in because the input data is multiplied with the CFO at the emitter of the desynchronized user $u$. Note that $\delta^{k(u)}=e^{j 2 \pi k \delta f^{(u)} / N_{p}}$, with $k=$ $0, \ldots, N_{p}+L_{c p}-2$, are the shift coefficients of user $u$.

\section{PROPOSED ESTIMATION ALGORITHM}

Given the model in eq.(4), we consider the joint maximum likelihood estimation of the channel and the CFO. Assuming the noise $n$ is uncorrelated and complex Gaussian with zero mean and variance $\sigma_{n}^{2}$, and the different users data are independent from each other, the ML estimate of the channel with respect to the $\mathrm{CFO}$ is given by

$$
\underline{\widehat{h}}^{(u)}=\left[\left(\underline{\delta}^{(u)} \odot \underline{\widetilde{X}}^{(u)}\right)^{H}\left(\underline{\delta}^{(u)} \odot \underline{\widetilde{X}}^{(u)}\right)\right]^{-1}\left(\underline{\delta}^{(u)} \odot \underline{\widetilde{X}}^{(u)}\right)^{H} \underline{r}
$$

The CFO estimate, $\delta \widehat{f}^{(u)}$, should be obtained by inserting $\underline{\widehat{h}}^{(u)}$ in to the log-likelihood function as follows

$$
\delta \widehat{f}=\arg \max _{\delta f}\left[J_{M L}(\delta f)\right]
$$

where the cost function is given by

$$
\begin{gathered}
J_{M L}(\delta f)=r^{H}\left(\underline{\delta}^{(u)} \odot \underline{\widetilde{X}}^{(u)}\right)\left[\left(\underline{\delta}^{(u)} \odot \underline{\widetilde{X}}^{(u)}\right)^{H}\left(\underline{\delta}^{(u)} \odot \underline{\tilde{X}}^{(u)}\right)\right]^{-1} \\
\left(\underline{\delta}^{(u)} \odot \underline{\widetilde{X}}^{(u)}\right)^{H} \underline{r}
\end{gathered}
$$

The cost function in eq.(7) is very complex to maximize because of the presence of the matrix inversion. By following the approach of [9] and choosing orthogonal training sequences like CHU-Codes [10], the cost function in eq.(7) can be simplified as

$$
J_{M L}(\delta f) \approx \underline{r}^{H}\left(\underline{\delta}^{(u)} \odot \underline{\widetilde{X}}^{(u)}\right)\left(\underline{\delta}^{(u)} \odot \underline{\widetilde{X}}^{(u)}\right)^{H} \underline{r}
$$

We propose to further simplify the above equation to

$$
J\left(\delta f^{(u)}\right)=\underline{r}^{H}\left(\underline{X}^{(u)} \odot \underline{\delta}_{h e r}^{(u)}\right) \underline{r}
$$

with

$$
\underline{X}^{(u)}=\underline{\widetilde{X}}^{(u)} \underline{\widetilde{X}}^{(u) H}
$$

and $\underline{\delta}_{h e r}^{(u)}$ is a hermitian matrix given by

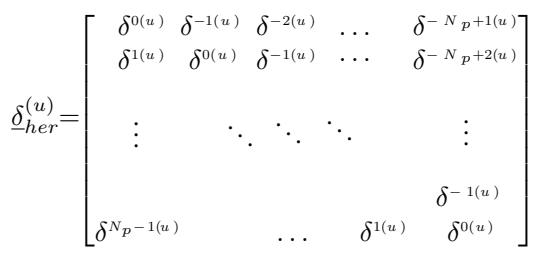


The high complexity of the grid search algorithms has led researchers to look for alternative ways to find estimation algorithms with low complexity to solve eq.(6). An alternative class of estimator proposed in [9, 11] uses polynomial approximations. For example in [11] authors have applied polynomial approximation to the cost function while in [9] the polynomial approximation of the shift matrix is applied. We propose to apply the polynomial approximation to the $\underline{\delta}_{h e r}^{(u)}$ matrix rather than the shift matrix $\underline{\delta}^{(u)}$ for user $u$. We found that the resulting estimator is simpler. We propose to approximate $\underline{\delta}_{h e r}^{(u)}$ as the first term of the following expression,

$$
\begin{aligned}
\underline{\delta}_{h e r}^{(u)} & =\sum_{m=0}^{\infty} \frac{\partial^{m}\left(\underline{\delta}_{h e r}^{(u)}\right)}{\partial \delta f^{(u) m}} \frac{\left(\delta f^{(u)}-\delta f_{o}^{(u)}\right)^{m}}{m !} \\
& =\sum_{m=0}^{\infty} \underline{D}^{m} \frac{\left(\delta f^{(u)}-\delta f_{o}^{(u)}\right)^{m}}{m !}
\end{aligned}
$$

where the matrix $\underline{D}^{m}$ is given by

$$
\underline{D}^{m}=\left(\frac{j 2 \pi}{N_{p}}\right)^{m}\left[\begin{array}{ccccc}
(0)^{m} & (-1)^{m} & (-2)^{m} & \ldots & \left(-N_{p}+1\right)^{m} \\
(1)^{m} & (0)^{m} & (-1)^{m} & \ldots & \left(-N_{p}+2\right)^{m} \\
(2)^{m} & (1)^{m} & (0)^{m} & \ddots & \\
\vdots & \ddots & \ddots & & \vdots \\
\left(N_{p}-2\right)^{m} & & & & \\
\left(N_{p}-1\right)^{m} & \left(N_{p}-2\right)^{m} & \ldots & & (0)^{m}
\end{array}\right] \underline{\delta}_{\text {he }} \delta f=\delta f_{o}
$$

Now eq.(9) becomes

$$
\begin{array}{r}
J\left(\delta f^{(u)}\right)=\underline{r}^{H}\left(\underline{X}^{(u)} \odot \sum_{m=0}^{\infty} \underline{D}^{m} \frac{\left(\delta f^{(u)}-\delta f_{o}^{(u)}\right)^{m}}{m !}\right) \underline{r} \\
=\sum_{m=0}^{\infty} \frac{\left(\delta f^{(u)}-\delta f_{o}^{(u)}\right)^{m}}{m !} \mathbb{R}\left\{\underline{r}^{H}\left(\underline{X}^{(u)} \odot \underline{D}^{m}\right) \underline{r}\right\}
\end{array}
$$

For a polynomial of degree $M$ expansion

$$
J\left(\delta f^{(u)}\right) \approx J_{\text {our }}\left(\delta f^{(u)}\right)
$$

with

$$
J_{\text {our }}\left(\delta f^{(u)}\right)=\sum_{m=0}^{M} \frac{\left(\delta f^{(u)}-\delta f_{o}^{(u)}\right)^{m}}{m !} \mathbb{R}\left\{\underline{r}^{H}\left(\underline{X}^{(u)} \odot \underline{D}^{m}\right) \underline{r}\right\}
$$

To find the frequency estimate, the first derivative of eq.(14) is set to zero

$$
\frac{\partial J_{o u r}\left(\delta f^{(u)}\right)}{\partial \delta f}=\sum_{m=0}^{M-1} \frac{\left(\delta f^{(u)}-\delta f_{o}^{(u)}\right)^{m}}{m !} d_{m+1}^{(u)}
$$

where the co-efficients $d_{m+1}^{(u)}$ are given by

$$
d_{m+1}^{(u)}=\mathbb{R}\left\{\underline{r}^{H}\left(\underline{X}^{(u)} \odot \underline{D}^{m+1}\right) \underline{r}\right\}
$$

From eq.(16) it can be seen that the co-efficients of the first derivative of the cost function $J_{\text {our }}\left(\delta f^{(u)}\right)$ are much simpler compared to those in [9]. For example, for a polynomial of degree $M=2$, the CFO estimator is given by

$$
\delta \widehat{f}^{(u)}=\delta f_{o}^{(u)}+\frac{-\mathbb{R}\left\{\underline{r}^{H}\left(\underline{X}^{(u)} \odot \underline{D}^{1}\right) \underline{r}\right\}}{\mathbb{R}\left\{\underline{r}^{H}\left(\underline{X}^{(u)} \odot \underline{D}^{2}\right) \underline{r}\right\}}
$$

where $\delta f_{o}^{(u)}$ is the initial guess of the CFO in the system.

\section{NUMERICAL RESULTS}

This section presents the simulations that are performed in order to validate our proposed estimation method.

We assume that there are two users in the system, $N_{u}=2$. The main simulation parameters considered are the total number of the available subcarriers $N_{p}=128$ and the length of the cyclic prefix is $L_{c p}=N_{p} / 8$. The channel is implemented with random impulse responses with Rayleigh fading coefficients and the length of the channel is equal to the length of the cyclic prefix $L_{h}=L_{c p}$. The orthogonal training sequences are generated using [10]. Initially the CFO experienced by both the users is taken as constant.

In Figure 1, we have plotted the mean and the variance of the $\delta \widehat{f}^{(u)}$ estimate about the true $\delta f^{(u)}$ value for iterations over different channel realizations. We can see that the esti-

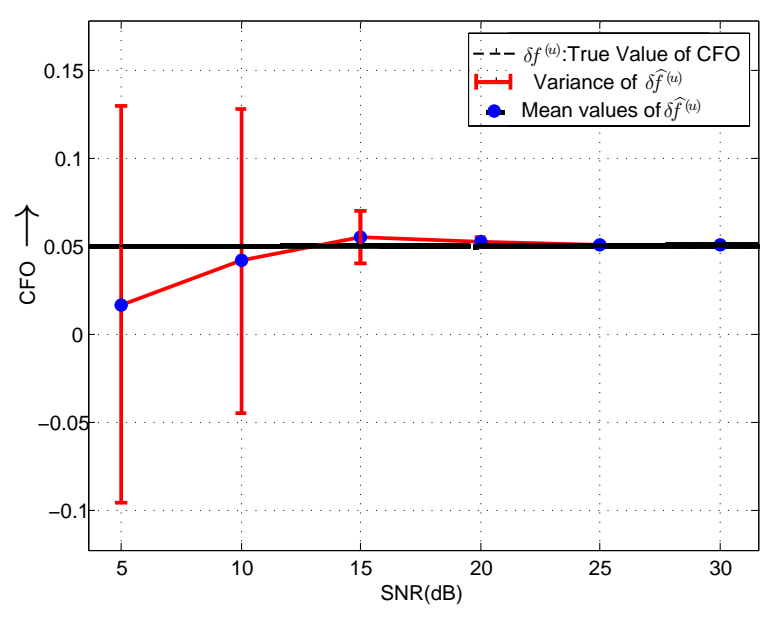

Fig. 1. Mean and variance of the CFO, $\delta \widehat{f}^{(u)}$ vs. SNR. 0.05 is the true value of the shift for user 1 , with $M=2, N_{p}=128, L_{c p}=16$ and $N_{u}=2$.

mated value approaches the true value with increase in SNR value. Thus for small values of the normalized CFO i.e. less than 0.1 , smaller values of $\mathrm{M}$ are sufficient to provide a closed form solution.

In Figure 2, we have plotted the mean and variance of the $\delta \widehat{f}^{(u)}$ estimate for a higher value of CFO $\delta f^{(u)}=0.3$. We 
used two polynomials of degree $M=2$ and $M=5$. The initial guess was taken equal to zero i.e $\delta \widehat{f}_{o}^{(u)}=0$ for $M=5$ while it was set deliberately equal to 0.15 for $M=2$ i.e. $\delta \widehat{f}_{o}^{(u)}=0.15$.

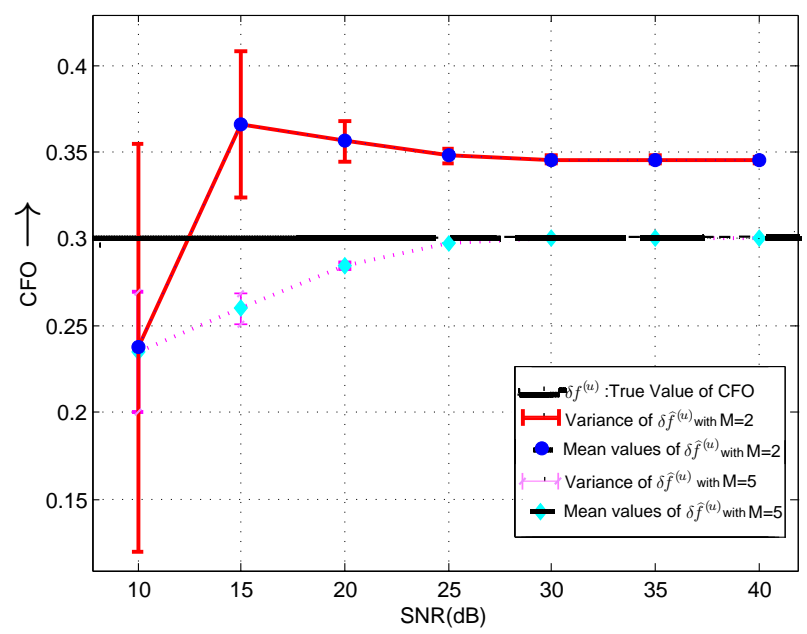

Fig. 2. Mean and variance of the CFO, $\delta \widehat{f}^{(u)}$ vs. SNR. 0.3 is the true value of the shift for user 1 , with $M=2, M=5, N_{p}=$ $128, L_{c p}=16$ and $N_{u}=2$.

For higher values of the CFO the $M=2$ estimator does not perform well because of the increased distance from the origin and the estimator does not converge unlike for $M=5$ which provides better results. So it can be seen that the degree of the polynomial determines the quality of the estimator. Our proposed model reduces the computational steps required in the calculation of the coefficients $d_{m+1}$ even more compared to the estimator method proposed in [9]. Calculation of $d_{m+1}$ involves simple Hadamard product which can be easily implemented. This difference comes into play because the estimator approach in [9] considers the CFO at the receiver only. Figure 3 shows the results for the mean squared error (MSE) values of the CFO along with the MSE values of estimated channel coefficients plotted against the SNR values for our proposed estimation method. Results in Figure 3 are computed with $M=3$. It is assumed that no initial knowledge of CFO is available i.e. $\delta f_{o}^{(u)}=0$. The CFO values of the two users are simulated as independent uniformly distributed random variables with a maximum possible value of $\delta f_{\max }=0.2$ i.e. for any user $u$ the CFO is $\delta f^{(u)} \leq \delta f_{\max }$.

\section{CONCLUSION}

In this paper, we have derived a new received signal model taking into account the $\mathrm{CFO}$ at the user terminal and before the multipath channel. We proposed a method for the joint estimation of the channel impulse response and the carrier frequency offsets for an uplink OFDMA system. Thanks to our signal model, we estimate the CFOs using a polynomial

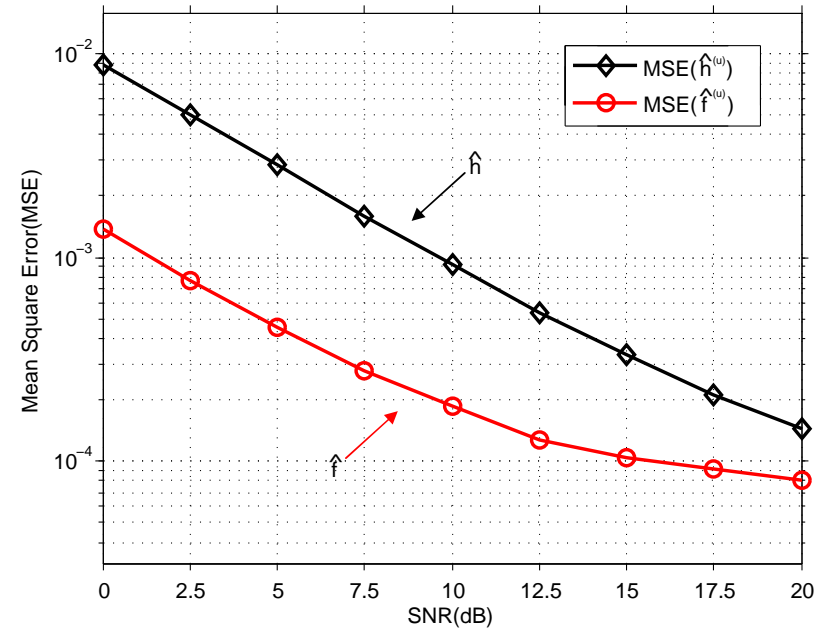

Fig. 3. MSE of $\widehat{h}_{l}^{(u)}$ and MSE of $\delta \widehat{f}^{(u)}$ vs the $\operatorname{SNR}(\mathrm{dB})$ for our proposed method with $M=3, N_{p}=128, L_{c p}=16$ and $N_{u}=2$ with independent uniformly distributed CFOs.

approximation. The CFO estimates are then used to estimate the channel using the maximum-likelihood approach.

\section{REFERENCES}

[1] A. F. Molisch, Wideband Wireless Digital Communication, Upper Saddle River: Prentice Hall, 2001.

[2] Qinfei Huang, Mounir Ghogho, Jibo Wei, and Philippe Ciblat, "Timing and frequency synchronization for OFDM based cooperative systems," ICASSP, IEEE International Conference on Acoustics, Speech and Signal Processing, pp. 26492652, 2009.

[3] B. Aziz, I. Fijalkow, and M. Ariaudo, "Intercarrier interference in uplink ofdma systems with carrier frequency offset," in IEEE PIMRC, Istambul, Turkey, September 2010 .

[4] Jan jaap Van De Beek, Ove Edfors, Magnus S, Sarah Kate Wilson, and Per Ola Brjesson, "On channel estimation in ofdm systems," in in Proc. of the IEEE Vehicular Technology Conference, VTC 95, 1995, pp. 815-819.

[5] P.H. Moose, "A technique for orthogonal frequency division multiplexing frequency offset correction," Communications, IEEE Transactions on, vol. 42, no. 10, pp. $2908-2914$, oct. 1994.

[6] M. Morelli, "Timing and frequency synchronization for the uplink of an ofdma system," Communications, IEEE Transactions on, vol. 52, no. 2, pp. 296 - 306, feb. 2004.

[7] J.-J. van de Beek, P.O. Borjesson, M.-L. Boucheret, D. Landstrom, J.M. Arenas, P. Odling, C. Ostberg, M. Wahlqvist, and S.K. Wilson, "A time and frequency synchronization scheme for multiuser ofdm," Selected Areas in Communications, IEEE Journal on, vol. 17, no. 11, pp. 1900 -1914, nov. 1999.

[8] M.-O. Pun, M. Morelli, and C.-C.J. Kuo, "Maximum-likelihood synchronization and channel estimation for ofdma uplink transmissions," Communications, IEEE Transactions on, vol. 54, no. 4, pp. 726 - 736, apr. 2006.

[9] L. Haring, S. Bieder, A. Czylwik, and T. Kaiser, "Estimation algorithms of multiple channels and carrier frequency offsets in application to multiuser ofdm systems," Wireless Communications, IEEE Transactions on, vol. 9, no. 3, pp. 865 -870 , mar. 2010 .

[10] D. Chu, "Polyphase codes with good periodic correlation properties (corresp.)," Information Theory, IEEE Transactions on, vol. 18, no. 4, pp. 531 - 532, jul. 1972.

[11] A. Abdel-Samad, "Fine frequency offset estimation for frequency-selective channels," may. 2006, vol. 4, pp. $1879-1883$. 